\title{
Symptom patterns and comparison of diagnostic methods in ragweed pollen allergy
}

\author{
LAURA HAIDAR $^{1-3}$, TUDOR-PAUL TAMAS ${ }^{1,2}$, FRANK STOLZ ${ }^{4}$, RAUL FLORIAN PETRISOR PATRASCU ${ }^{1}$, \\ KUAN-WEI CHEN ${ }^{2}$ and CARMEN PANAITESCU ${ }^{1-3}$
}

\author{
${ }^{1}$ Discipline of Physiology, Department III Functional Sciences, 'Victor Babes' University of Medicine and \\ Pharmacy, 300041 Timisoara; ${ }^{2}$ Centre for Gene and Cellular Therapies in The Treatment of Cancer-OncoGen, \\ 'Pius Brinzeu' Clinical Emergency Hospital, 300723 Timisoara; ${ }^{3}$ Center of Immuno-Physiology \\ and Biotechnologies, 'Victor Babes' University of Medicine and Pharmacy, 300041 Timisoara, \\ Romania; ${ }^{4}$ Department of Product Development, Biomay AG, A-1090 Vienna, Austria
}

Received December 31, 2020; Accepted February 2, 2021

DOI: $10.3892 /$ etm.2021.9957

\begin{abstract}
The aim of the present study was to determine the pattern of symptoms of ragweed pollen-induced allergic disease in sensitized patients from Romania and to compare the molecular diagnosis of allergy with the skin prick test, in order to better characterize allergic patients and to guide therapy. A total of 97 subjects, including patients with ragweed pollen-induced allergic rhinoconjunctivitis with/without asthma, as well as healthy controls, were recruited prospectively in one ragweed pollen season, submitted to allergy questionnaires, skin prick tests and multiplex specific IgE (immunoglobulin E) measurement by ImmunoCAP ISAC (ImmunoCAP Immuno-Solid phase Allergy Chip) assay. A total of 83 patients were sensitized to ragweed pollen. Most patients $(73 \%)$ were diagnosed with moderate-severe intermittent allergic rhinoconjunctivitis and $25 \%$ of the patients also had allergic asthma. The most common symptoms were watery rhinorrhea $(91.57 \%)$, nasal obstruction $(86.75 \%)$, and sneezing (85.54\%). Most patients were polysensitized $(62.65 \%)$, especially to other pollens, house dust mites and animal danders. Only $90 \%$ of the patients with positive
\end{abstract}

Correspondence to: Professor Carmen Panaitescu, Discipline of Physiology, Department III Functional Sciences, 'Victor Babes' University of Medicine and Pharmacy, Timisoara, 2 Eftimie Murgu Square, 300041 Timisoara, Romania

E-mail: cbunu@umft.ro

Abbreviations: A. artemisiifolia, Ambrosia artemisiifolia; ALEX ${ }^{2}$, Allergy explorer; ARIA, Allergic Rhinitis and its Impact on Asthma; AIT, allergen immunotherapy; GA ${ }^{2}$ LEN, Global Allergy and Asthma European Network; GINA, Global Initiative for Asthma; HDM, house dust mites; IgE, immunoglobulin E; ImmunoCAP ISAC, ImmunoCAP Immuno-Solid phase Allergy Chip; ISAC standard units, ISU; SPT, skin prick test

Key words: ragweed pollen allergy, skin prick test, ImmunoCAP ISAC assay, IgE, molecular diagnosis, allergic rhinoconjunctivitis skin prick test to ragweed pollen extract also had increased specific serum IgE to Amb a 1. Current options for specific molecular diagnosis of ragweed allergy are limited, as they only contain one or few of the sensitizing allergens present in ragweed pollen. An improved component-resolved diagnosis, using several ragweed pollen allergens, is required for better patient characterization and subsequent selection of an appropriate allergen immunotherapy product, thereby enabling a more personalized approach to the management of the ragweed-allergic patient.

\section{Introduction}

Allergic rhinoconjunctivitis is an IgE (immunoglobulin E)-mediated disorder that affects the nasal mucosa and the conjunctiva. Its prevalence in adults varies within Europe, ranging from $17 \%$ (in Italy) to $29 \%$ (Belgium) (1,2). Inhaled allergens are a significant factor in morbidity and may severely affect quality of life (3-5). Symptoms of allergic rhinoconjunctivitis include sneezing, nasal itching and obstruction, watery nasal discharge, eye itching and congestion, and tearing, while coughing episodes, difficulty breathing or wheezing are associated with asthma. Major causative agents for allergic rhinoconjunctivitis are pollen grains, house dust mites, pet danders and fungal spores $(6,7)$.

Over the last few decades, a matter of particular concern in this field has been Ambrosia (ragweed) pollen, its presence in the air being caused by the plants dispersing it and meteorological processes that alter pollen release, dissemination, transport or deposition on surfaces (8).

Ragweed (family Asteraceae, genus Ambrosia) is an invasive species of annual herbaceous flowering plant, originally native to Northern America, which started to spread through Europe in the 19th century (9). This weed can often be found on roadsides, riverbanks, or abandoned lands and fields. Ragweed seeds are able to survive for many decades in the soil and the plant grows again under favorable conditions (10). Climate change (particularly rising temperatures and carbon dioxide levels), urbanization and pollution have led to the increase in ragweed biomass, length of the pollen season and atmospheric 
pollen counts, while current forecasts show an upward trend in the future (11). These factors play and will continue to play a major role in the increasing burden generated by ragweed sensitization for people living in affected areas (12).

The most prevalent species is Ambrosia artemisiifolia (common or short ragweed), which is also clinically the most relevant for its high potential to cause allergic sensitization. Ragweed pollen can be transported over great distances, while trade and traffic globalization have been identified as the most important factors for ragweed distribution. Ragweed pollen has been identified at great distances, of more than $600 \mathrm{~km}$ offshore and over $3 \mathrm{~km}$ up in the air (13). A single ragweed plant is estimated to produce one billion pollen grains during one season $(14,15)$. The symptoms induced by ragweed pollen in sensitized individuals correlate with and may last beyond the pollen season. In Romania, only partial statistics regarding ragweed pollen spread and the number of ragweed-allergic patients are available $(16,17)$. The highest atmospheric ragweed pollen concentration is encountered in August and September, but the pollen is present in the air for a much longer period, starting with mid-July and up to late October, depending on weather conditions (18). There are currently 11 recognized ragweed pollen allergens, numbered from 1 to 11 (Amb a 2 was renamed as isoform Amb a 1.05), out of which Amb a 1 and Amb a 11 are considered major allergens, inducing sensitization in more than 50\% of ragweed-allergic patients (19). Moreover, some ragweed pollen allergens have homology to pollen allergens from Artemisia vulgaris (mugwort), for example, Amb a 1 and Art v 6, Amb a 4 and Art v 1 (20), raising the question of whether some patients are truly co-sensitized to both pollens, or whether cross-reactivity is at play (21). Mugwort belongs to the plant family Asteraceae; its pollen season is longer than that of ragweed, with which it partly overlaps. However, mugwort pollen concentrations in the atmosphere do not reach such high values as ragweed, therefore it does not affect as great a number of individuals.

The diagnosis of allergic rhinoconjunctivitis and asthma relies on the precise medical history of the patient, especially regarding potential sources of exposure, seasonal symptoms of nasal inflammation and airway hyperresponsiveness. The main clinical test used in practice is the skin prick test (SPT), which can be accompanied by other diagnostic tools such as nasal allergen challenge or spirometry. Paraclinical testing includes molecular diagnosis and allows the identification of sensitizing allergens and cross-reactions by measuring specific serum IgE to relevant allergens. SPT is usually considered the first line of diagnosis of IgE-mediated allergies, due to its advantages over specific serum $\operatorname{IgE}$, such as rapid results, flexibility, low cost and good tolerability. The sensitivities and specificities of the two methods have been compared in studies, with varying results, some showing that SPT is more sensitive and less specific $(22,23)$, while others show the complete opposite $(24,25)$. Precise diagnosis is necessary for adequate management of allergic rhinitis, which may involve allergen immunotherapy (AIT).

The aim of the present study was to determine the symptom pattern of ragweed pollen-induced allergic disease on sensitized patients from Western Romania, to investigate potential cross-sensitivities or co-sensitivities with other allergens, and to compare the molecular diagnosis of allergy by specific $\operatorname{IgE}$ measurement with the SPT, with the goal of improving allergic patient characterization and therapeutic guidance.

\section{Patients and methods}

Ethics approval and consent to participate. All SPTs were performed and all peripheral blood samples were obtained from the participants after the signing of the informed consent elaborated under an approved protocol by the Ethics in Scientific Research Commission of the 'Pius Brinzeu' County Clinical Emergency Hospital Timisoara, which complies with Romanian laws (Law no. 95/2006, article 67, and article 28, chapter VIII 904/2006) and with EU GCP Directive 2005/28/EC (26), International Conference of Harmonisation of Technical Requirements for Registration of Pharmaceuticals for Human Use (ICH) (27) and the Declaration of Helsinki-Recommendations Guiding Medical Doctors in Biomedical Research Involving Human Subjects (28).

Study design and patients. A total of 83 ragweed allergic patients and 14 heathy controls (97 subjects in total) were recruited and observed prospectively in one ragweed pollen season (August, 2018 to November, 2018), in a cross-sectional study. The clinical research was performed in allergy clinics from Timisoara, the main town from the Western part of Romania. The study included patients that during ragweed pollen season presented rhinitis, with or without conjunctivitis, diagnosed according to ARIA (Allergic Rhinitis and its Impact on Asthma) criteria (29), with or without asthma, diagnosed according to GINA (Global Initiative for Asthma) criteria (30), and that also had a positive result on the SPT with ragweed pollen extract. Patients with chronic pathologies such as cancer and autoimmune disease were excluded, as well as those with histamine skin wheals on SPT of less than $2 \mathrm{~mm}$. Negative controls, without any allergic symptoms and with negative SPT were also included. The healthy controls were recruited to ensure that the SPT technique was correct and that the allergen extracts were not providing false-positive results, in order to consider the SPT as the gold standard; these subjects were not included in the further analyses.

Symptom evaluation. A self-reported symptom evaluation score was also applied (Appendix S1). Briefly, the patients were asked to rate the intensity of their current, or, in case they were already under antiallergic therapy, their maximal symptoms, on a scale from 0 to 10 . ' 0 ' was defined as asymptomatic, and ' 10 ' as intense symptoms that greatly interfere with daily activities, including sleep, thereby making them impossible. A symptom score, calculated by summing up all the symptoms pertaining to allergy that a patient presents, was also evaluated.

Allergen extracts. Patient evaluation was performed by SPT to a panel of 19 extracts of inhaled allergens (HAL, Düsseldorf, Germany), containing standardized cutaneous extracts for prick tests: Hazel (Corylus avellana), alder (Alnus incana), birch (Betula alba), plane (Platanus vulgaris), oak (Quercus robur), grass mix (Poa pratensis, Dactilis glomerata, Lolium perenne, Phleum pratense, Festuca pratensis, Helictotrichon pretense), cereal mix (Triticum aestivum, Hordeum vulgare, Secale cereale), mugwort (Artemisia vulgaris), ragweed 
(Ambrosia artemisiifolia), fungi (Alternaria alternata, Cladosporium herbarum, Aspergillus fumigatus tested separately), yeasts (Candida albicans, Saccharomyces mellis tested separately), dog (Canis familiaris), cat (Felis catus), house dust mites (Dermatophagoides pteronyssinus, Dermatophagoides farinae tested separately), and cockroach (Blatella germanica). Histamine dihydrochloride $(10 \mathrm{mg} / \mathrm{ml}$, equivalent to $6 \mathrm{mg}$ histamine) was used as the positive control, and a phenolated glycero-saline solution as the negative control. The panel of allergen extracts was selected according to the recommendation of the Global Allergy and Asthma European Network $\left(\mathrm{GA}^{2} \mathrm{LEN}\right)$ for a common panel of allergens for Europe (31); however, it did not include cypress and olive tree pollens, nor Parietaria pollen, as these plant species are not commonly encountered in Romania.

Allergy skin prick tests. Allergy SPT was performed according to the $\mathrm{GA}^{2} \mathrm{LEN}$ recommendation for harmonization of skin prick testing (32). Briefly, drops of allergen extract were applied on the volar aspect of the forearm with a distance of at least $2 \mathrm{~cm}$ between them and then the skin was pricked with lancets. Mean wheal diameter was recorded after $15 \mathrm{~min}$ and a wheal diameter of at least $3 \mathrm{~mm}$ was considered a positive SPT. The tests were performed by trained specialists on healthy skin, and after appropriate withdrawal of treatments that may interfere with the skin reaction, such as antihistamines, long-term or high-dose glucocorticoids, antidepressants, or omalizumab. The following notations were made, according to wheal mean diameter: $>3$ to $<4 \mathrm{~mm}$, ' + ' (very mildly reactive), $\geq 4$ to $<10 \mathrm{~mm}$, ' ++ ' (mildly reactive), $\geq 10$ to $<15$, ' +++ ' (moderately reactive) and $\geq 15$, '++++' (very reactive), and the presence of pseudopodia was also recorded. This classification of the skin response was used in order to better correlate it with the IgE class.

ImmunoCAP ISAC assay. For the evaluation of allergenspecific IgE antibodies in blood, the ImmunoCAP ISAC (Immuno-Solid phase Allergy Chip) assay was used, which is a semi-quantitative molecular diagnostic test that reports results in ISAC standard units (ISU), indicating allergen-specific IgE levels; the operating range is 0.3-100 ISU. The specific ISAC microarray chip that was used was developed through the European Union-funded project Mechanisms for the Development of Allergies; (MeDALL) (33), which can measure IgE antibodies to 176 allergen components (34), using only $20 \mu \mathrm{l}$ of serum. A sample consisting of $5 \mathrm{ml}$ of venous blood was collected from each patient in a red-top blood collection tube (without anticoagulant or preservative). After collection of the whole blood, it was allowed to clot by leaving it undisturbed at room temperature for $30 \mathrm{~min}$, and then the clot was removed by centrifugation at $1,500 \mathrm{x} g$ for $10 \mathrm{~min}$ at room temperature. The resulting supernatant was immediately apportioned into $0.5 \mathrm{ml}$ aliquots and stored at $-70^{\circ} \mathrm{C}$ until further processing. Allergen-specific $\operatorname{IgE}$ was measured in sera from ragweed-allergic patients using a fluoroenzyme immunoassay auto-analyser (Thermo Fisher Scientific Inc., Phadia AB), according to the manufacturer's guidelines. Briefly, $20 \mu \mathrm{l}$ of serum was added to each microarray and incubated at room temperature for $120 \mathrm{~min}$. The samples were washed and then incubated for $30 \mathrm{~min}$ with $20 \mu \mathrm{l}$ of fluorescence-labeled antihuman IgE antibodies. Unbound antibodies were removed by washing and the fluorescence of the processed ISAC slides was measured in a GenePix4000B microarray scanner (Molecular Devices). Image analysis was performed using a microarray image analyzer software (MIA, Thermo Fisher Scientific Inc., Phadia AB). Fluorescence measurements were compared with a calibration curve and expressed as ISU. Established cut-off values were used to interpret the results: Values $<0.3$ ISU were considered negative (undetectable or very low) with regards to sensitization to a specific allergen, and values of $\geq 0.3$ ISU were distributed into three classes, as follows: For ISU values $\geq 0.3$ to $<1$, class 1 (low); for ISU values $\geq 1$ to $<15$, class 2 (moderate to high); and for ISU values $\geq 15$, class 3 (very high). IgE class according to ISU was also correlated with the intensity of the response to ragweed pollen extract in the SPT. The chip included only the established major allergen from ragweed pollen, Amb a 1, and not other ragweed pollen allergens, nor ragweed pollen extract. The sensitivity and specificity of ImmunoCAP ISAC were compared against the SPT, which was considered the standard method for allergy diagnosis, as it was the method used for patient selection.

Statistical analysis. Statistical analysis and data collection were performed using Microsoft Office Excel 2013, two-tailed tests and non-parametric tests such as Spearman's rho to measure the strength of association between two variables. A statistical significance threshold of 0.05 was used $(\mathrm{P}<0.05)$.

\section{Results}

Demographical data, clinical symptoms, and skin prick test results. A total of 83 ragweed-allergic patients were recruited in the study, according to their symptoms and the results of the SPT to ragweed pollen extract. Mean patient age was $31.2 \pm 8.9$ years, with a mean allergic disease age (duration since first appearance of symptoms) of $3.62 \pm 3.15$ years. A total of $40 \%$ of the patients were male, and $60 \%$ female. There were no significant differences between male and female patients regarding skin sensitivity. Most patients (73\%) were diagnosed with moderate-severe intermittent allergic rhinoconjunctivitis, and $25 \%$ of the patients also had allergic asthma. Mean maximum intensity of symptoms was 8.15 out of 10 on the self-evaluation scale. The most common symptoms were: Watery rhinorrhea (in $91.57 \%$ of the patients), nasal obstruction (in $86.75 \%$ of the patients), sneezing (in $85.54 \%$ of the patients), nasal pruritus (in $75.90 \%$ of the patients), and eye pruritus (in $75.90 \%$ of the patients) (Fig. 1).

The majority of the patients were polysensitized (62.65\%) according to the SPT results. Most of the polysensitized patients were sensitized to other pollens (cereals, grasses, Artemisia, birch, oak), but a large percentage was also sensitized to house dust mites (HDM), the main perennial allergen in this geographical area (Fig. 2).

All patients with a disease history of more than 10 years had developed allergic asthma, with exacerbations during the ragweed pollen season. (Fig. 3A). However, when looking separately at patients only sensitized to ragweed pollen vs. patients also sensitized to house dust mites (HDM), only 


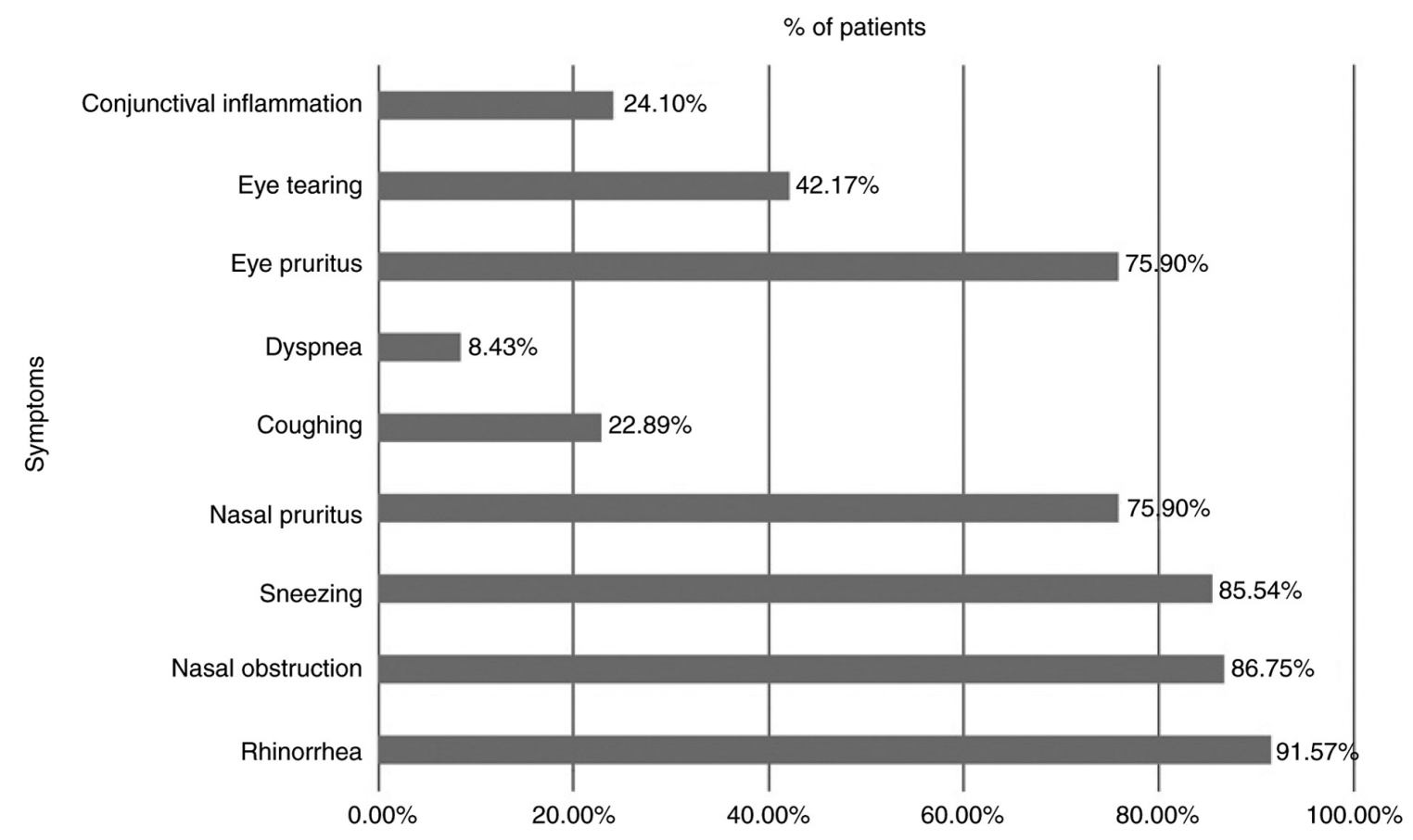

Figure 1. Symptom distribution of ragweed-allergic patients.

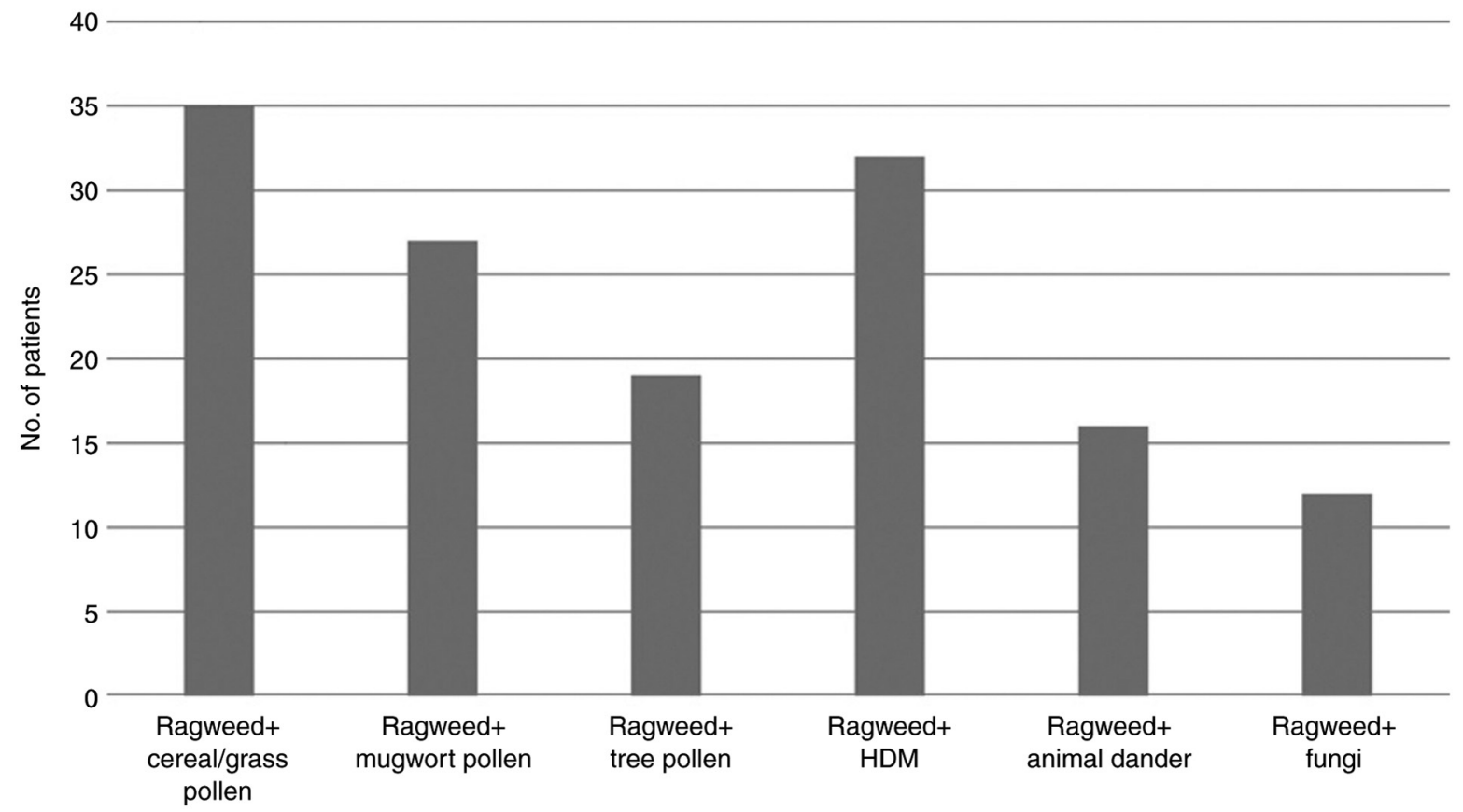

Figure 2. Distribution of monosensitized/polysensitized patients. HDM, house dust mite.

the HDM-sensitized patient group had a disease history of $>10$ years (Fig. 3 B).

Correlation between self-reported evaluation score and a computed symptoms score. A moderate correlation was found between the self-reported evaluation score (on a scale 0-10) and a computed symptoms score (by adding up all the symptoms a patient presents), by using Spearman's rho $(0.584)$, which was extremely significant $(\mathrm{P}<0.0001)$, indicating an accurate correlation between the patients' estimate of disease severity and the number of symptoms (Fig. 4). However, the $\mathrm{R}^{2}$ obtained was 0.25 , which only partially explains the variability of the response data around its mean. This may occur because only the number of symptoms present in a patient was taken into account, not also the severity of each symptom. Therefore, there could be patients with only one, very severe symptom, significantly affecting their quality of life, or patients with several mild symptoms that do not significantly interfere with their daily activities. 
A

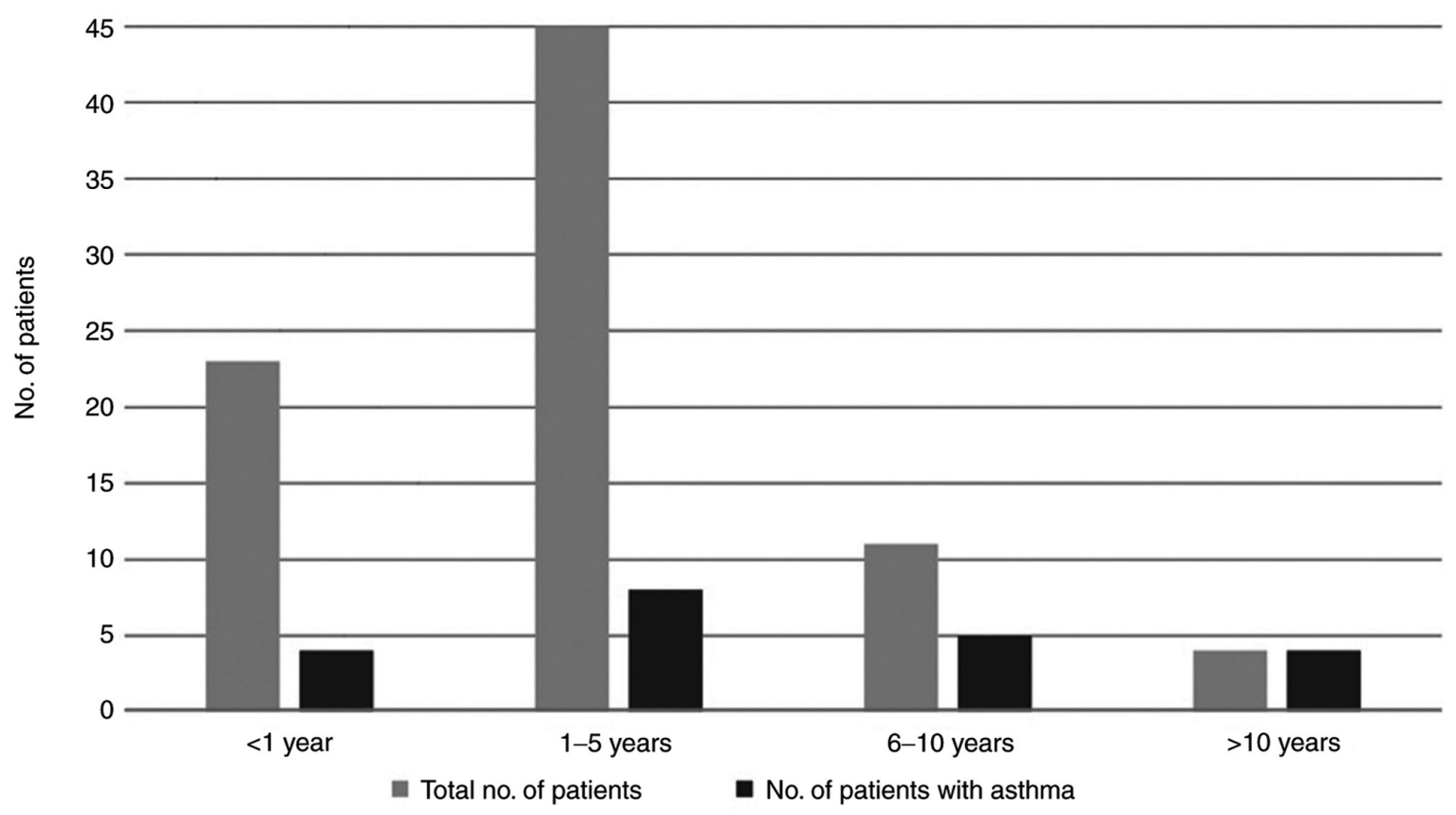

B

60

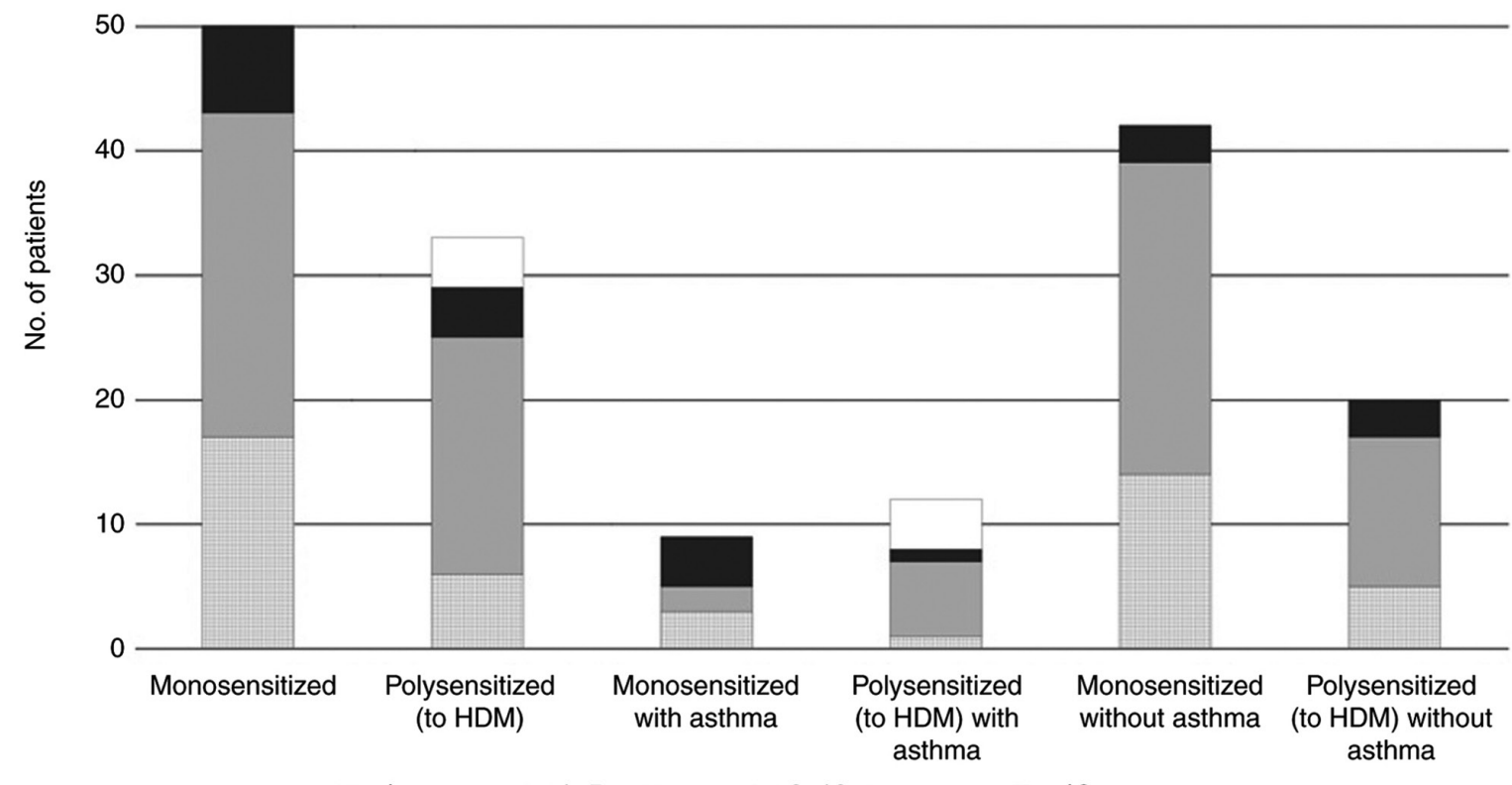

Figure 3. Asthma development in ragweed-allergic patients. (A) Progression of allergic inflammation to asthma. (B) Progression of allergic inflammation to asthma, separated into monosensitized vs. polysensitized. In this figure, 'polysensitized' refers to patients who were sensitized to ragweed pollen and to HDM concomitantly. HDM, house dust mite.

ImmunoCAP ISAC assay results. The results obtained in vivo, by SPT, were compared against the immunoarray chip, which measures semi-quantitatively specific serum $\operatorname{IgE}$ against 176 molecular allergens. It includes several molecular allergens from sources such as HDM (Dermatophagoides pteronyssinus and farinae), timothy grass (Phleum pratense), or dog (Canis familiaris), but only one allergen from ragweed. It does not include any allergens from cultivated grasses or cereal pollens, such as rye, wheat, or barley, nor from oak tree, which induced sensitizations in some of the patients included in the study, as measured by SPT. Significantly, it includes several mold allergens, but none of the patients sensitized to molds, as determined by SPT, had a positive result for specific serum IgE against molds (Fig. 5, Table I).

Out of the 83 patients with positive SPT to ragweed pollen extract, $90 \%$ also had increased specific serum IgE 


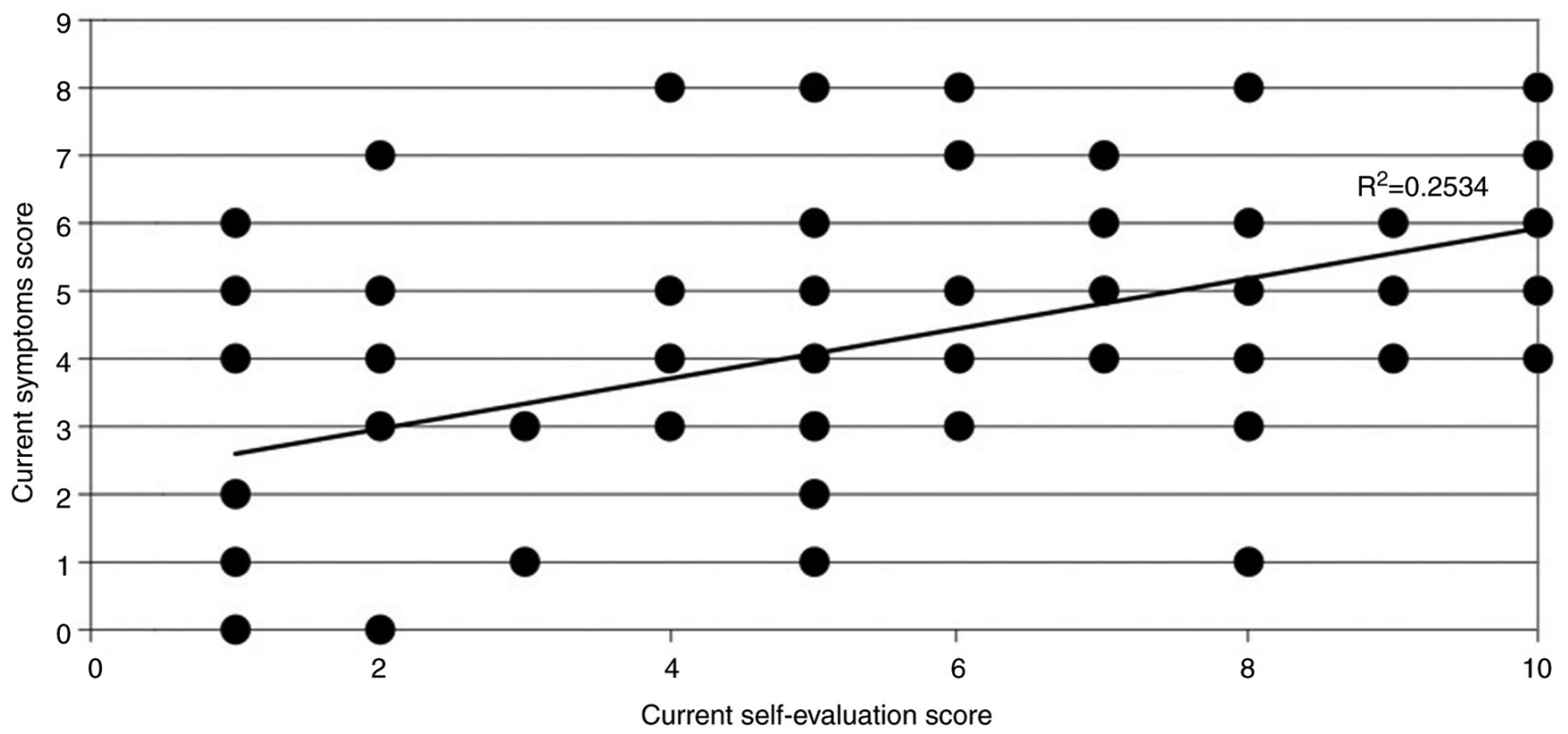

Figure 4. Correlation between self-reported evaluation score and computed symptoms score.

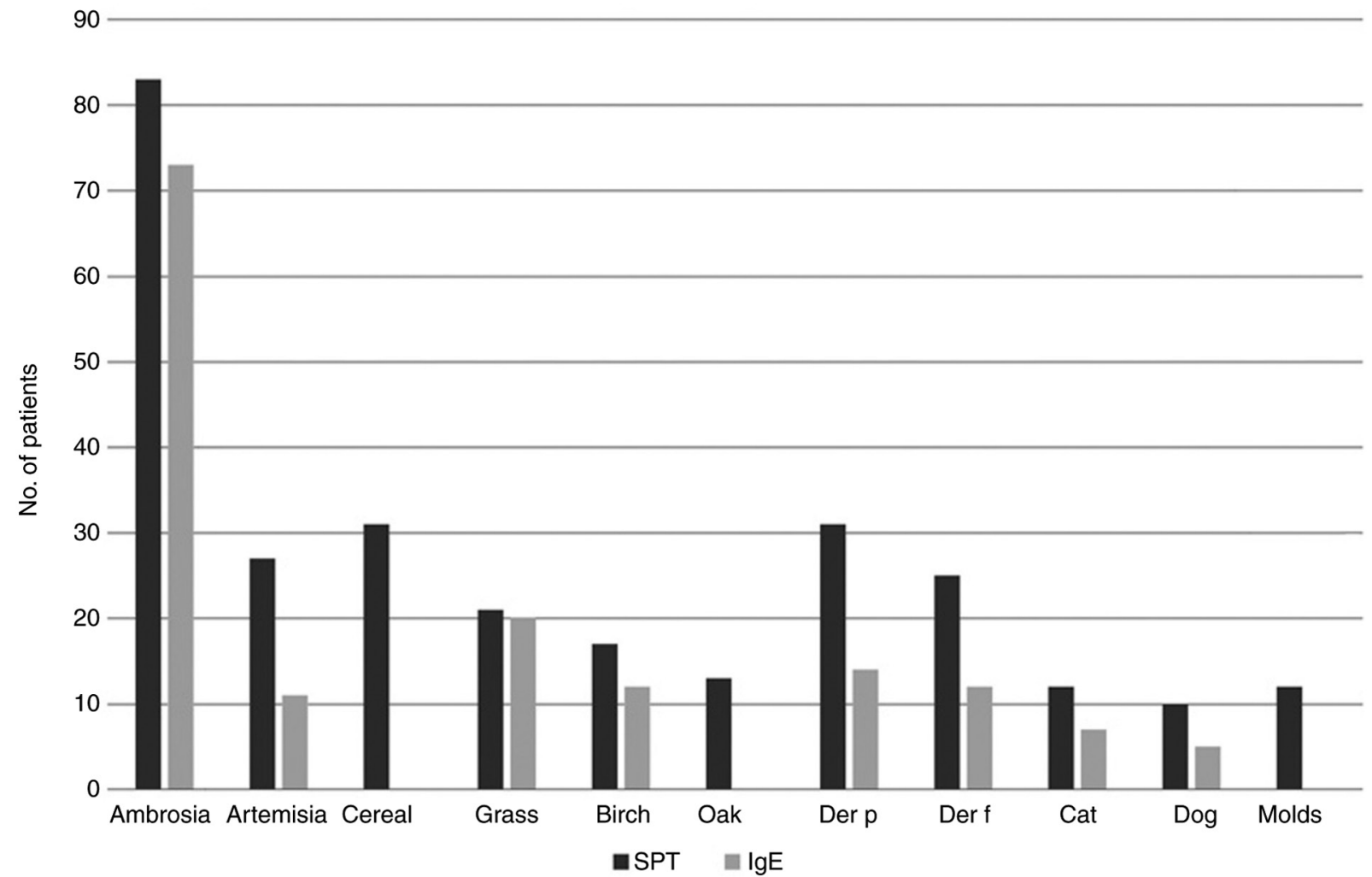

Figure 5. Comparison between SPT and IgE results. SPT, skin prick test; IgE, immunoglobulin E. Der p, Dermatophagoides pteronyssinus; Der f, Dermatophagoides farinae.

levels to the major ragweed allergen, Amb a $1(5 \%$ class 1 , $35 \%$ class 2 , and $45 \%$ class 3 ). Sensitivity and specificity of the ImmunoCAP ISAC microarray (Amb a 1) vs. SPT with ragweed pollen extract was $85.88 \%$ (95\% CI: 76.63-92.48\%), and $90.91 \%$ (95\% CI: 58.67-98.49\%), respectively. However, $\operatorname{IgE}$ class did not correlate with the mean diameter of the wheal in SPT.
A particular situation was noted regarding the 11 patients that had a positive SPT to ragweed pollen extract and negative specific IgE to Amb a 1. The possibility of cross-reactivity with allergens from mugwort pollen was examined in more detail, due to the high homology shared by several allergens from these two plants from the Asteraceae family, and a possible interpretation is provided in Table II. 
Table I. Comparison between SPT and IgE results.

\begin{tabular}{lccccccccccc}
\hline & Ambrosia & Artemisia & Cereal & Grass & Birch & Oak & Der $\mathrm{p}$ & Derf & Cat & Dog & Molds \\
\hline $\begin{array}{l}\text { \% positive IgE out of } \\
\text { positive SPT }\end{array}$ & 87.95 & 40.74 & 0 & 95.23 & 70.58 & 0 & 45.16 & 48 & 58.33 & 50 & 0 \\
\hline
\end{tabular}

SPT, skin prick test; IgE, immunoglobulin E; Der p, Dermatophagoides pteronyssinus; Der f, Dermatophagoides farinae.

Table II. Interpretation of SPT to ragweed pollen extract and mugwort pollen extract, and specific IgE to Amb a 1 and Art v 1.

\begin{tabular}{|c|c|c|}
\hline Results & No. of patients & Interpretation \\
\hline $\begin{array}{l}\text { SPT to mugwort neg and } \\
\text { Art v } 1 \text { neg }\end{array}$ & 7 & $\begin{array}{l}\text { Probably sensitized to other major and/or minor allergens from ragweed } \\
\text { pollen, but which do not share cross-reactivity with allergens from } \\
\text { mugwort (e.g., Amb a 11) }\end{array}$ \\
\hline $\begin{array}{l}\text { SPT to mugwort neg and } \\
\text { Art v } 1 \text { pos }\end{array}$ & 2 & Probably due to cross-sensitization between Art v 1 and Amb a 4 \\
\hline $\begin{array}{l}\text { SPT to mugwort pos and } \\
\text { Art v } 1 \text { neg }\end{array}$ & 1 & $\begin{array}{l}\text { Probably sensitized to minor allergens from ragweed pollen which have } \\
\text { cross-reactivity with minor allergens from mugwort pollen (e.g., } \\
\text { profilins-Amb a } 8 \text { with Art v 4, or polcalcins-Amb a } 9 \text { with Art v 5) }\end{array}$ \\
\hline $\begin{array}{l}\text { SPT to mugwort pos and } \\
\text { Art v } 1 \text { pos }\end{array}$ & 1 & Probably sensitized to mugwort pollen \\
\hline
\end{tabular}

SPT, skin prick test; IgE, immunoglobulin E; pos, positive; neg, negative.

Potential cross-reactivity between Amb a 1 and other allergens from same pectate lyase family, but from species not present in the local flora, such as Cry j 1 from Japanese cedar and Cup a 1 from Arizona cypress was also investigated. Cry j 1 specific IgE were identified in $24 \%$ of the Amb a 1 positive patients, as opposed to none of the Amb a 1 negative patients, which was a statistically significant difference $(\mathrm{P}<0.05)$. Cup a 1 specific IgE was identified in $\sim 8 \%$ of the Amb a 1 positive patients, as opposed to none of the Amb a 1 negative patients, which was statistically insignificant $(\mathrm{P}>0.05)$ (Table III).

\section{Discussion}

The principle of the skin prick test (SPT) is that the introduction of relevant allergens in the skin induces a wheal and flare response, which can be measured in a standardized fashion. This reaction is due to the cross-linkage of specific IgE bound on mast cells, which leads to their degranulation and subsequent release of inflammatory mediators, such as histamine (35). However, in vitro methods have become an important complementary tool for allergy diagnosis, and they are even starting to replace the in vivo diagnosis, due to its limitations. SPT has several contraindications, for example it cannot be used in patients with extensive eczema, dermographism, urticaria with or without angioedema (36), or in patients under medication that may interfere with the test results, such as antihistamines, glucocorticoids, and some classes of antidepressants (31). Another limitation is the fact that allergen extracts are biological mixtures, containing different
Table III. Cross-reactivity between Amb a 1 and Cry j 1, and Cup a 1 respectively.

\begin{tabular}{lcc}
\hline & \multicolumn{2}{c}{ SPT ragweed pos } \\
\cline { 2 - 3 } & Amb a 1 neg (\%) & Amb a 1 pos (\%) \\
\hline Cry j 1 neg & 100.00 & 76.06 \\
Cry j 1 pos & 0.00 & 23.94 \\
Cup a 1 neg & 100.00 & 91.55 \\
Cup a 1 pos & 0.00 & 8.45 \\
\hline
\end{tabular}

SPT, skin prick test; pos, positive; neg, negative.

concentrations of allergens, depending on the extraction method; therefore, results obtained with allergen extracts from different manufacturers can vary greatly $(37,38)$. These variations are even more relevant when the biological extracts are used for allergen immunotherapy (39). Currently, the numbers of available extracts for in vivo testing and for immunotherapy has diminished greatly, due to the increasingly stringent rules for market approval in Europe (40). Another point that has not been addressed is the potential of SPT to induce de novo sensitizations, as the skin has been known to be an effective route for sensitization $(41,42)$.

Despite all these limitations, the advantages of the SPT such as its cost efficiency, extensive support by national health insurance houses, and lack of expensive equipment 
requirements make it an indispensable tool for the immediate diagnosis of allergic patients by physicians of different specialties, and enhance patient compliance by providing a convincing visual image of the extent of their sensitivities.

Unlike allergen extracts and natural allergens, recombinant allergens produced by techniques of molecular engineering are pure, and produced under standardized, reproducible conditions. They can be further used for singleplex (ImmunoCAP) or multiplex (ISAC microarray chip, $\mathrm{ALEX}^{2}$ ) molecular diagnosis of respiratory and food allergies, as well as for allergen immunotherapy. Molecular allergy diagnosis can differentiate between genuine sensitization and cross-reactivity, as well as guide allergen immunotherapy to a more targeted and personalized approach (43). ImmunoCAP ISAC, based on 112 different molecular components, is the most studied and most frequently used molecular diagnostic tool based on a microarray. Yet, in the present study we used an experimental microarray chip with 176 allergens, and technically, chips with many more allergens can be blotted as well as personalized chips with different allergen patterns, according to patient symptoms and medical history. Currently microarray chips are not used as the primary diagnostic tool, due to their high costs; moreover, they are also not covered by national health insurance. Unfortunately, socioeconomic status influences healthcare.

Any result, either obtained by in vivo or in vitro methods, should be interpreted in the context of clinical symptoms and medical history, and, as using either method by itself may lead to inappropriate diagnosis of some patients, it is therefore recommended to use the in vivo and in vitro testing methods in complementary fashion whenever possible. When the test results and the medical history are inconclusive, challenge tests may help in determining the clinical relevance of sensitization.

The two major allergens from ragweed pollen are considered to be Amb a 1, a pectate lyase, and Amb a 11, a cysteine protease (44). SPT extracts may contain variable amounts of these proteins and also of the other allergenic proteins from ragweed pollen, depending on the extraction methods as well as on the local characteristics of the plants (45). However, to date, only methods of testing for specific IgE against ragweed pollen extract, Amb a 1 and Amb a 4 exist. When using the ImmunoCAP ISAC microarray chip, which only includes Amb a 1, not all sera from patients that tested positive to ragweed pollen extract by SPT showed a positive reaction. This suggests that some ragweed-allergic patients would be undiagnosed or misdiagnosed by using current in vitro diagnosis methods, which rely on component-resolved diagnosis, but do not have the full array of allergens from ragweed pollen. As shown in Table II, these patients may fall under several different scenarios-either sensitized primarily to other major and/or minor allergens from ragweed pollen, some of which may be shared with mugwort or with other plants, or they may be sensitized primarily to other plants, which contain pan-allergens such as profilins or polcalcins sharing homology with ragweed allergens. Therefore, they may also not respond to allergen immunotherapy (AIT) using recombinant Amb a 1, as they are likely sensitized to Amb a 11 or minor allergens from ragweed pollen.

The identification of Cry $\mathrm{j} 1$-specific $\operatorname{IgE}$ in patients sensitized to Amb a 1 is interesting, as the sequence identity between the molecules is not too high, 46-49\% (46), even though both, as well as Cup a 1, are pectate lyases. In addition, exposure to Japanese cedar was minimal in this sample population, as it is not a local plant.

The clinical utility of a precise molecular diagnosis in allergic diseases was demonstrated in a previous study by Chen et al (47), who performed a post hoc analysis of sera from house dust mite (HDM)-allergic patients who had been treated by subcutaneous HDM AIT in a double-blind, placebo-controlled clinical study, regarding their IgE and IgG reactivity against a comprehensive panel of HDM allergens by ImmunoCAP ISAC technology. The clinical effects of AIT had been evaluated in the patients by controlled allergen exposure in the Vienna Challenge Chamber. HDM extracts used for allergy diagnosis with SPT and AIT vary regarding allergen concentration, as they are only standardized for Der $\mathrm{p} 1$ and Der $\mathrm{p} 2$, even though there are several other clinically relevant allergens, such as Der p 5, Der p 7, Der p 21, and Der p 23. The study showed that only anti-Der $\mathrm{p} 1$ and anti-Der $\mathrm{p} 2$ IgG levels increased in patients that had undergone AIT with HDM extract. Patients that had also been sensitized to other HDM allergens beside Der $\mathrm{p} 1$ and Der $\mathrm{p} 2$ showed no signs of clinical improvement after AIT. Similar outcomes are expected regarding ragweed-allergic patients undergoing AIT; therefore, a precise diagnosis regarding the sensitization pattern is required. Molecular diagnosis using all clinically relevant ragweed pollen allergens could offer support to allergists, for identification of several categories of patients: i) Those who may benefit from AIT with extracts currently available from companies, ii) those who may be treated with Amb a 1-only AIT, and iii) those who may need a more personalized approach with a specific pattern of clinically relevant allergens.

In conclusion, the present study showed that a small fraction of ragweed-allergic patients, which are sensitized only to minor ragweed pollen allergens and/or to Amb a 11, cannot be identified with standard in vitro diagnostic procedures. Therefore, the development of an improved component-resolved diagnosis, using several ragweed pollen allergens, is required for a better patient characterization and subsequent selection of an appropriate AIT product. The improvement of component-resolved diagnosis will allow for a more personalized approach to the management of the ragweed-allergic patient, consisting in an AIT product that contains only the allergens relevant to a particular patient, thereby ensuring better patient outcomes.

\section{Acknowledgements}

Not applicable.

\section{Funding}

This work was supported through the project 'INnovative Strategies for Prevention, diagnosis and therapy of ragweed pollen Induced REspiratory Diseases' (INSPIRED), MySMIS 103663, COP 2014-2020 92/09.09.2016, funded by the National Authority for Scientific Research and Innovation (ANCSI), under the Operational Program Competitiveness 2014-2020. 


\section{Availability of data and materials}

The datasets used and/or analyzed during the current study are available from the corresponding author on reasonable request.

\section{Authors' contributions}

LH conceptualized and visualized the experiments, developed the methodology, and prepared the original draft of the manuscript. LH and TPT performed the formal analysis, validated and interpreted the data. $\mathrm{LH}$ and $\mathrm{CP}$ supervised the study. LH, TPT and CP performed the investigations. LH, FS, RFPP, KWC and CP reviewed the data, revised the manuscript and edited the final manuscript. All authors have read and agreed to the published version of the manuscript.

\section{Ethics approval and consent to participate}

All skin prick tests were performed and all peripheral blood samples were obtained after signing the informed consent elaborated under an approved protocol by the Ethics in Scientific Research Commission of the 'Pius Brinzeu' Clinical Emergency County Hospital Timisoara, which complies with Romanian laws (Law no. 95/2006, article 67, and article 28, chapter VIII 904/2006) and with EU GCP Directive 2005/28/EC, International Conference of Harmonisation of Technical Requirements for Registration of Pharmaceuticals for Human Use (ICH) and the Declaration of Helsinki-Recommendations Guiding Medical Doctors in Biomedical Research Involving Human Subjects.

\section{Patient consent for publication}

Not applicable.

\section{Competing interests}

The authors declare no competing interests. The funders had no role in the design of the study; in the collection, analyses, or interpretation of data; in the writing of the manuscript, or in the decision to publish the results.

\section{Authors' information}

ORCiD: Laura Haidar: https://orcid.org/0000-0002-5703-2578; Carmen Panaitescu: https://orcid.org/0000-0001-8749-9972.

\section{References}

1. Bauchau V and Durham SR: Epidemiological characterization of the intermittent and persistent types of allergic rhinitis. Allergy 60: 350-353, 2005.

2. D'Amato G, Cecchi L, Bonini S, Nunes C, Annesi-Maesano I, Behrendt H, Liccardi G, Popov T and Van Cauwenberge P: Allergenic pollen and pollen allergy in Europe. Allergy 62: 976-990, 2007.

3. Pawankar R, Canonica GW, Holgate ST, Lockey RF and Blaiss M: The World Allergy Organization (WAO) White Book on Allergy: Update 2013. World Allergy Organization (WAO), Milwaukee, WI, pp87-92, 2013.

4. Bumbacea RS, Corcea SL, Ali S, Dinica LC, Fanfaret IS and Boda D: Mite allergy and atopic dermatitis: Is there a clear link? (Review). Exp Ther Med 20: 3554-3560, 2020.
5. Solomon I, Ilie MA, Draghici C, Voiculescu VM, Caruntu C, Boda D and Zurac S: The impact of lifestyle factors on evolution of atopic dermatitis: An alternative approach. Exp Ther Med 17: 1078-1084, 2019

6. Singh AB and Mathur C: An aerobiological perspective in allergy and asthma. Asia Pac Allergy 2: 210-222, 2012.

7. Bumbacea R, Berghea E and Giurcaneanu C: Frequency of contact sensitisation in children with atopic dermatitis. Allergy 62 (Suppl 83): S319, 2007.

8. Šaulienè I and Veriankaitè L: Analysis of high allergenicity airborne pollen dispersion: Common ragweed study case in Lithuania. Ann Agric Environ Med 19: 451-455, 2012.

9. Smith M, Cecchi L, Skjøth CA, Karrer G and Šikoparija B: Common ragweed: A threat to environmental health in Europe. Environ Int 61: 115-126, 2013.

10. Buters J, Alberternst B, Nawrath S, Wimmer M, Traidl-Hoffmann C, Starfinger U, Behrendt H, Schmidt-Weber C and Bergmann KC: Ambrosia artemisiifolia (ragweed) in Germany-current presence, allergological relevance and containment procedures. Allergo J Int 24: 108-120, 2015.

11. Ziska LH, Gebhard DE, Frenz DA, Faulkner S, Singer BD and Straka JG: Cities as harbingers of climate change: Common ragweed, urbanization, and public health. J Allergy Clin Immunol 111: 290-295, 2003.

12. Oswalt ML and Marshall GD: Ragweed as an example of worldwide allergen expansion. Allergy Asthma Clin Immunol 4: 130-135, 2008.

13. Smith M, Jäger S, Berger U, Šikoparija B, Hallsdottir M, Saulienė I, Bergmann KC, Pashley CL, de Weger L, Majkow ska-Wojciechowska B, et al: Geographic and temporal variations in pollen exposure across Europe. Allergy 69: 913-923, 2004.

14. Fumanal B, Chauvel B and Bretagnolle F: Estimation of pollen and seed production of common ragweed in France. Ann Agric Environ Med 14: 233-236, 2007.

15. Bullock JM, Chapman D, Schafer S, Roy D, Girardello M, Haynes T, Beal S, Wheeler B, Dickie I, Phang Z, et al: Assessing and controlling the spread and the effects of common ragweed in Europe. Final Report to the European Commission, DG Environment. 2012. Available online: https://ec.europa. eu/environment/nature/invasivealien/docs/Final_Final_Report. pdf. Accessed June 18, 2020).

16. Leru PM, Matei D and Ianovici N: Health impact of Ambrosia artemisiifolia reflected by allergists practice in Romania. A questionnaire-based survey. Ann West Univ Timisoara Series Biol 18: 43-54, 2015.

17. Popescu FD and Tudose AM: Ambrosia pollen sensitization in allergic rhinitis patients from the central part of the Romanian Plain. Romanian J Rhinol 1: 26-30, 2011.

18. Ianovici N, Panaitescu CB and Brudiu I: Analysis of airborne allergenic pollen spectrum for 2009 in Timişoara, Romania. Aerobiologia (Bologna) 29: 95-111, 2013.

19. Chen KW, Marusciac L, Tamas PT, Valenta R and Panaitescu C: Ragweed pollen allergy: Burden, characteristics, and management of an imported allergen source in Europe. Int Arch Allergy Immunol 176: 163-180, 2018.

20. Pichler U, Hauser M, Wolf M, Bernardi ML, Gadermaier G, Weiss R, Ebner C, Yokoi H, Takai T, Didierlaurent A, et al: Pectate lyase pollen allergens: Sensitization profiles and cross-reactivity pattern. PLoS One 10: e0120038, 2015.

21. Asero R, Bellotto E, Ghiani A, Aina R, Villalta D and Citterio S: Concomitant sensitization to ragweed and mugwort pollen: Who is who in clinical allergy? Ann Allergy Asthma Immunol 113: 307-313, 2014.

22. Kianifar HR, Pourreza A, Azad FJ, Yousefzadeh H and Masomi F: Sensitivity comparison of the skin prick test and serum and fecal radio allergosorbent test (RAST) in diagnosis of food allergy in children. Rep Biochem Mol Biol 4: 98-103, 2016.

23. Bignardi D, Comite P, Mori I, Ferrero F, Fontana V, Bruzzone M, Mussap M and Ciprandi G: Allergen-specific IgE: Comparison between skin prick-test and serum assay in real-life. Allergol Select 40: 16-22, 2017.

24. Asha'ari ZA, Suhaimi Y, Yusof RA, Rushdan I and Maraina CH: Comparison of serum specific IgE with skin prick test in the diagnosis of allergy in Malaysia. Med J Malaysia 66: 202-206, 2011.

25. Kumar R, Gupta N, Kanuga J and Kanuga M: A comparative study of skin prick test versus serum-specific IgE measurement in Indian patients with bronchial asthma and allergic rhinitis. Indian J Chest Dis Allied Sci 57: 81-85, 2015. 
26. Verheugen G: Commission directive 2005/28/EC laying down principles and guidelines for good clinical practice as regards investigational medicinal products for human use, as well as the requirements for authorization of the manufacturing or importation of such products. Official J Eur Union 13: 91, 2005.

27. International Council on Harmonisation of Technical Requirements for Registration of Pharmaceuticals for Human Use (ICH). https://www.ema.europa.eu/en/ partners-networks/international-activities/multilateral-organisationsinitiatives/international-council-harmonisation-technical-requirementsregistration-pharmaceuticals-human-use\#ich-guidelines-andtechnical-requirements-section. Accessed June 18, 2020).

28. World Medical Association declaration of Helsinki. Recommendations guiding physicians in biomedical research involving human subjects. JAMA 277: 925-926, 1997.

29. Brożek JL, Bousquet J, Agache I, Agarwal A, Bachert C, Bosnic-Anticevich S, Brignardello-Petersen R, Canonica GW, Casale T, Chavannes $\mathrm{NH}$, et al: Allergic rhinitis and its impact on Asthma (ARIA) guidelines-2016 revision. J Allergy Clin Immunol 140: 950-958, 2017.

30. Global Initiative for Asthma. Global Strategy for Asthma Management and Prevention, 2018. https://ginasthma. org/wp-content/uploads/2019/01/2018-GINA.pdf. Accessed June 18, 2020)

31. Heinzerling L, Mari A, Bergmann KC, Bresciani M, Burbach G, Darsow U, Durham S, Fokkens W, Gjomarkaj M, Haahtela T, et al: The skin prick test-European standards. Clin Transl Allergy 3: 3, 2013.

32. Heinzerling LM, Burbach GJ, Edenharter G, Bachert C, Bindslev-Jensen C, Bonini S, Bousquet J, Bousquet-Rouanet L, Bousquet PJ, Bresciani M, et al: GA(2)LEN skin test study I: GA(2)LEN harmonization of skin prick testing: Novel sensitization patterns for inhalant allergens in Europe. Allergy 64: 1498-1506, 2009.

33. Mechanisms for the Development of Allergies (MeDALL). https://cordis.europa.eu/project/id/261357. Accessed June 18, 2020.

34. Lupinek C, Wollmann E, Baar A, Banerjee S, Breiteneder H, Broecker BM, Bublin M, Curin M, Flicker S, Garmatiuk T, et al: Advances in allergen-microarray technology for diagnosis and monitoring of allergy: The MeDALL allergen-chip. Methods 66: 106-119, 2014

35. Kowal K, DuBuske L and Wood RA: Overview of skin testing for allergic disease. UpToDate, Waltham, MA. https://www.uptodate.com/contents/overview-of-skin-testing-forallergic-disease?search=Overview\%20 of $\% 20$ skin $\% 20$ testing $\% 20$ for $\% 20$ allergic $\% 20$ disease $\&$ source $=$ search_result\&selectedTitle $=$ $1 \sim 150 \&$ usage_type $=$ default\&display_rank $=1$. Accessed June 18 , 2020.

36. Leru PM, Anton VF, Bocsan C, Muntean A and Boda D: Acquired angioedema induced by angiotensin-converting enzyme inhibitors-experience of a hospital-based allergy center. Exp Ther Med 20: 68-72, 2020.
37. Focke M, Marth K and Valenta R: Molecular composition and biological activity of commercial birch pollen allergen extracts. Eur J Clin Invest 39: 429-436, 2009.

38. González-Pérez R, Poza-Guedes P, del Pino YB, Matheu V and Sánchez-Machín I: Evaluation of major mite allergens from European standardized commercial extracts for in vivo diagnosis: Addressing the need for precision medicine. Clin Transl Allergy 9: 14-21, 2019.

39. Moreno Benítez F, Espinazo Romeu M, Letrán Camacho A, Mas S, García-Cózar FJ and Tabar AI: Variation in allergen content in sublingual allergen immunotherapy with house dust mites. Allergy 70: 1413-1420, 2015.

40. Valenta R, Karaulov A, Niederberger V, Zhernov Y, Elisyutina O, Campana R, Focke-Tejkl M, Curin M, Namazova-Baranova L, Wang JY, et al: Allergen extracts for in vivo diagnosis and treatment of allergy: Is there a future? J Allergy Clin Immunol Pract 6: 1845-1855.e2, 2018.

41. Jensen-Jarolim E, Jensen AN and Canonica GW: Debates in allergy medicine: Molecular allergy diagnosis with ISAC will replace screenings by skin prick test in the future. World Allergy Organ J 10: 33-38, 2017.

42. Agache I, Bilò M, Braunstahl GJ, Delgado L, Demoly P, Eigenmann P, Gevaert P, Gomes E, Hellings P, Horak F, et al: In vivo diagnosis of allergic diseases-allergen provocation tests. Allergy 70: 355-365, 2015.

43. van Hage $M$, Hamsten $C$ and Valenta R: ImmunoCAP assays: Pros and cons in allergology. J Allergy Clin Immunol 140: 974-977, 2017.

44. Bouley J, Groeme R, Le Mignon M, Jain K, Chabre H, Bordas-Le Floch V, Couret MN, Bussieres L, Lautrette A, Naveau M, et al: Identification of the cysteine protease Amb a 11 as a novel major allergen from short ragweed. J Allergy Clin Immunol 136: 1055-1064, 2015.

45. Pagani M, Antico A, Cilia M, Calabro D, Poto S, Pecora S and Burastero SE: Comparison of different diagnostic products for skin Prick testing. Eur Ann Allergy Clin Immunol 41:23-31, 2009.

46. Mohapatra SS, Lockey RF and Polo F: Weed Pollen Allergens. In: Allergens and Allergen Immunotherapy, 4th edition. Lockey RF and Ledford DK (eds). Informa Healthcare, New York, NY, pp127-140, 2008.

47. Chen KW, Zieglmayer P, Zieglmayer R, Lemell P, Horak F, Bunu CP, Valenta R and Vrtala S: Selection of house dust mite-allergic patients by molecular diagnosis may enhance success of specific immunotherapy. J Allergy Clin Immunol 143: $1248-1252,2019$

This work is licensed under a Creative Commons Attribution-NonCommercial-NoDerivatives 4.0 International (CC BY-NC-ND 4.0) License. 